TITLE:

\title{
Microcoils and microsamples in solid-state NMR.
}

$\operatorname{AUTHOR}(\mathrm{S})$ :

Takeda, Kazuyuki

\section{CITATION:}

Takeda, Kazuyuki. Microcoils and microsamples in solid-state NMR..

Solid state nuclear magnetic resonance 2012, 47-48: 1-9

ISSUE DATE:

2012-10

URL:

http://hdl.handle.net/2433/189441

\section{RIGHT:}

(c) 2012 Elsevier Inc.; This is not the published version. Please cite only the published version.; この論文は出版社版でありません。引用の際に は出版社版をご確認ご利用ください。 


\title{
Microcoils and microsamples in solid-state
}

\section{NMR}

\author{
Kazuyuki Takeda ${ }^{\mathrm{a}, *}$, \\ ${ }^{a}$ Division of Chemistry, Graduate School of Science, Kyoto University, 606-8502 \\ Kyoto, Japan
}

\begin{abstract}
Recent reports on microcoils are reviewed. The first part of the article includes a discussion of how the geometries of the sample and coil affect the NMR signal intensity. In addition to derivation of the well-known result that the signal intensity increases as the coil size decreases, the prediction that dilution of a small sample with magnetically inert matter leads to better sensitivity if a tiny coil is not available is given. The second part of the review focuses on the issues specific to solid-state NMR. They include realization of magic-angle spinning (MAS) using a microcoil and harnessing of such strong pulses that are feasible only with a microcoil. Two strategies for microcoil MAS, the piggyback method and magic-angle coil spinning (MACS), are reviewed. In addition, MAS of flat, disk-shaped samples is discussed in the context of solid-state NMR of small-volume samples. Strong RF irradiation, which has been exploited in wide-line spectral excitation, multiple-quantum MAS (MQMAS), and dipolar decoupling experiments, has been accompanied by new challenges regarding the Bloch-Siegert effect, the minimum time resolution of the spectrometer, and the time scale of pulse transient effects. For a possible solution to the latter problem, recent reports on active compensation of pulse transients are described.
\end{abstract}


Key words: microcoil, piggyback microcoil MAS, magic-angle coil spinning (MACS), disk MAS, strong RF irradiation, active compensation of transients

\section{Introduction}

A tiny NMR sample coil with a volume of the order of or less than a microliter is known as a microcoil [1]. The microcoil was originally used in liquid-state NMR to increase the sensitivity for small samples [2]. Since then, the microcoil has been applied to solid-state NMR in various circumstances. The purpose of this review is to focus on research on solid-state NMR using a microcoil, to discuss its innovative aspects and problems that have arisen, and to suggest future directions of development for further progress in microcoil solid-state NMR.

In the first part of this review, we discuss the intensity of signals induced by spatially distributed nuclear magnetization. The signal-to-noise ratio in NMR and microcoil NMR is of interest in both liquid- and solid-state NMR, and has already been studied extensively [3]. Here, we focus on how the coil and sample geometries affect the NMR signal intensity.

In addition, we address the following issues that are specific to solids. First, magic angle spinning (MAS) is an indispensable strategy for high-resolution experiments. Thus, the MAS-microcoil combination is quite appealing. Studies on microcoil MAS reported so far are divided into two groups. One uses a sample capillary sticking out of the conventional rotor, while the other spins

* corresponding author Email address: takezo@kuchem.kyoto-u.ac.jp (Kazuyuki Takeda). 
a microresonator together with the sample. Both of these interesting schemes, together with a strategy for MAS of flat, small-volume samples such as thin films and deposited layers, are reviewed.

Second, microcoils have another attractive feature in that much stronger RF irradiation is applicable than previously feasible. This feature could be harnessed to expand the arena of solid-state NMR. Relevant topics are wideline excitation, spin decoupling, and multiple-quantum (MQ) excitation and conversion. As discussed below, however, realization of strong irradiation has brought other challenges to be overcome in the future.

\section{Intensity of nuclear induction signals}

The term filling factor is frequently used as a measure of the fraction of the coil volume occupied by the sample. In general, larger filling factors yield better sensitivity. The reason why the sensitivity increases with the filling factor may be explained by the reciprocity theorem, which states that the RF circuit that gives the highest RF field per unit current results in the best sensitivity. In the following, we analyze the relationship between the filling factor and the sensitivity without using the reciprocity theorem to provide an intuitive account of the reason why the NMR signal intensity can be enhanced by reducing the coil size.

Consider nuclear magnetization $\mathbf{M}$ at the center of a solenoid coil of diameter $D$ (radius $R=D / 2$ ), length $L$, and $n$ turns. The coil axis is tilted by angle $\theta$ with respect to the external field. For static samples, the natural choice would be $\theta=\pi / 2$, or $\theta=\cos ^{-1}(1 / \sqrt{3})$ for MAS. First, we assume that the sample is 
infinitesimally small. The finite size case is dealt with below. When a $\pi / 2$ pulse is applied to the spin system in thermal equilibrium, the magnetization starts to precess around the static field $B_{0}$ at the Larmor frequency $\omega_{0}=-\gamma B_{0}$, where $\gamma$ is the gyromagnetic ratio. As a result, electromotive force (EMF) $\xi$ is induced across the coil terminals. According to the Faraday law, $\xi$ is given by the time derivative of the magnetic flux $\Phi$ penetrating through the path of the coil wire.

Since $\mathbf{M}$ is precessing at $\omega_{0}$, the flux $\Phi$ oscillates, i.e.,

$$
\Phi(t)=\Phi_{0} \sin \theta \cos \left(\omega_{0} t+\varphi\right) .
$$

The amplitude $\Phi_{0}$ is given by the magnetic flux at the moment at which the magnetization vector $\mathbf{M}$ is parallel to the coil axis. $\Phi_{0}$ is represented as

$$
\Phi_{0}=\int_{\text {loop }} \mathbf{B} \cdot \mathbf{n} d a
$$

where $\mathbf{B}(t)$ is the magnetic field produced by $\mathbf{M}, d a$ is an infinitesimal area inside the loop, and $\mathbf{n}$ is a unit vector normal to $d a$.

As shown in Fig. 1, the magnetic field lines exit from the magnetization, turn around, and eventually return to where they started. It is important to note that not all magnetic field lines contribute to the EMF. That is, the lines that return inside the loop, as depicted in Fig. 1 by the broken lines, result in no net magnetic flux and thereby zero EMF. Note that the fraction of the EMFactive parts of the field lines taking a path outside the loop can be increased by reducing the size of the loop. This is why the smaller the coil is, the better is the sensitivity for a given fixed magnetization. 
Using the Stokes theorem, Eq. (2) can be rewritten as

$$
\Phi_{0}=\oint_{\text {loop }} \mathbf{A} \cdot d \mathbf{s}
$$

where $\mathbf{A}(t)$ is the vector potential due to $\mathbf{M}$ and the integral is taken along the path of the wire. At position $\mathbf{r}, \mathbf{A}(\mathbf{r})$ produced by $\mathbf{M}$ is given by $\mathbf{A}(\mathbf{r})=$ $\left(\mu_{0} / 4 \pi\right)(\mathbf{M} \times \mathbf{r}) / r^{3}$. Then $\Phi_{0}$ is calculated as

$$
\begin{aligned}
\Phi_{0} & =\oint \mathbf{A} \cdot d \mathbf{s}=\frac{\mu_{0}}{4 \pi} M R^{2} \int_{-n \pi}^{+n \pi} d \alpha\left[R^{2}+\left(\frac{\alpha L}{2 \pi n}\right)^{2}\right]^{-\frac{3}{2}} \\
& =\frac{\mu_{0} M n}{\sqrt{L^{2}+D^{2}}} .
\end{aligned}
$$

The simplest coil configuration, albeit not practical, is the case of $n=1$ and $L=0$, which corresponds to a loop coil, giving

$$
\Phi_{0}=\frac{\mu_{0} M}{D}
$$

That is, the EMF is inversely proportional to the coil diameter $D$, as stated in literature.

Next, we consider the case of a sample of finite size. For simplicity, we assume that the sample is a flat disk with radius $a$ over which the nuclear spins are homogeneously distributed. The sample is placed inside a single-turn coil, as described in Fig. 2. Using net nuclear magnetization $M$, the magnetization $m$ per unit surface is given by

$$
m=\frac{M}{\pi a^{2}}
$$

The magnetic flux $\Phi_{0}^{\prime}$ due to nuclear spins in an infinitesimal element $r d \phi d r$ is represented as 


$$
\begin{aligned}
\Phi_{0}^{\prime} & =\oint_{\text {loop }} d \mathbf{s} \cdot \mathbf{A} \\
& =\frac{\mu_{0}}{4 \pi} m \operatorname{Rr} d \phi d r \int_{0}^{2 \pi} d \alpha \frac{\cos \beta}{b^{2}} .
\end{aligned}
$$

Using $b^{2}=R^{2}+r^{2}-2 r R \cos \alpha$ and $\cos \beta=(R-r \cos \alpha) / b$, Eq. (9) is rewritten as

$$
\Phi_{0}^{\prime}=\frac{\mu_{0}}{4 \pi} m R r d \phi d r \int_{0}^{2 \pi} d \alpha(R-r \cos \alpha)\left(R^{2}+r^{2}-2 r R \cos \alpha\right)^{-\frac{3}{2}} .
$$

This integration can be performed analytically:

$$
\Phi_{0}^{\prime}=\frac{\mu_{0}}{4 \pi} \frac{M}{\pi a^{2}} r d \phi d r\left\{\frac{2}{R-r} K\left[-\frac{4 r R}{(R-r)^{2}}\right]+\frac{2}{R+r} E\left[-\frac{4 r R}{(R-r)^{2}}\right]\right\}
$$

Here, $K$ and $E$ are complete elliptic integrals of the first and second type, respectively.

To evaluate the contribution of nuclear magnetization over the sample volume, we expand Eq. (11) into a Taylor series as

$$
\Phi_{0}^{\prime}=\frac{\mu_{0}}{4 \pi} \frac{M d \phi d r}{a^{2}}\left[\frac{2 r}{R}+\frac{3 r^{3}}{2 R^{3}}+\frac{45 r^{5}}{32 R^{5}}+\frac{175 r^{7}}{128 R^{7}}+\frac{11025 r^{9}}{8192 R^{9}}+\frac{43659 r^{11}}{32768 R^{11}}+\cdots\right] \text {, }
$$

from which we obtain

$$
\begin{aligned}
\Phi_{0} & =\int_{0}^{a} d r \int_{0}^{2 \pi} d \phi \Phi_{0}^{\prime} \\
& =\frac{\mu_{0} M}{D}\left[1+\frac{3}{8} \frac{a^{2}}{R^{2}}+\frac{15}{64} \frac{a^{4}}{R^{4}}+\frac{175}{1024} \frac{a^{6}}{R^{6}}+\frac{2205}{16384} \frac{a^{8}}{R^{8}}+\frac{14553}{131072} \frac{a^{10}}{R^{10}}+\cdot(1 \beta)\right.
\end{aligned}
$$

The first term of Eq. (13) indicates that the EMF is inversely proportional to the coil diameter, as is also the case for point magnetization. In addition, a gain due to the higher-order terms would be expected, which is negligibly small when $a \ll R$ but becomes considerable as the ratio $a / R$ approaches to unity. Fig. 3 shows how the amplitude $\Phi_{0}$ of the magnetic flux, normalized 
by $\mu_{0} M / D$, varies with $a / R$. It is clear that the enhancement factor increases with the ratio, giving an approximately two-fold gain when $a=0.95 R$. This result points to a second choice for dealing with tiny samples when a microcoil is not available. That is, a gain in signal intensity can be obtained by diluting the material of interest with magnetically inert media so that the sample just fits inside the regular coil.

It should be noted that the above prediction is based on a rather simplified assumption of a single-loop coil and a disk-shaped sample with no thickness. The quantitative behavior of the EMF for realistic situations would be more complicated. In addition, the gain in EMF is obtained at the cost of degrading the RF homogeneity over the sample. This would cause a non-uniform excitation profile for the nuclear spins. Moreover, the sensitivity is determined not only by the EMF but also by the quality factor $(Q)$ of the tank circuit, which depends on the coil geometry in a complicated way, as discussed extensively in the literature [3-5]. Nevertheless, the analysis given here is useful, as it helps in understanding the relationship between the signal intensity and the sample and coil geometries. It is also worth pointing out that the widely used term EMF is confusing, as it is not a force but a potential.

\section{Fabrication of microcoils}

The traditional way of hand-winding microcoils may be possible for coil diameters down to $\sim 100 \mu \mathrm{m}$. However, accurate control of the coil shape in a reproducible manner would be a formidable task. Alternatively, a coil-winding apparatus would be helpful [6,7]. In addition, coil fabrication by lithographic means [8-13] is of interest, although most of these reports concern liquid-state 
NMR.

Demas et al. studied the performance of lithographically produced microcoils and wire-wound microcoils of similar geometries [12]. The cross-section of the former is rectangular. The wire width and coil pitch can be controlled accurately and arbitrarily, while the maximum thickness of the wire is currently limited to ca. $20 \mu \mathrm{m}$ for technical reasons. By contrast, wire-wound microcoils have a round cross-section and the diameter of commercially available wire is discrete $(25,50,100, \ldots \mu \mathrm{m})$. For common coil dimensions (pitch, number of turns, wire diameter or width), Demas et al. reported that a wire-wound coil resulted in a higher $Q$ factor compared to lithographic coils [12]. This was ascribed simply to the larger cross-section of the wound coil wire, giving less AC resistance. When the authors compared lithographic and wire-wound coils with equal cross-sectional areas, they found that the overall performances were comparable. Therefore, lithographic schemes would be of interest for tiny coils for which hand winding would be formidable and/or when conducting wire of a desired diameter is not available.

To accommodate diverse samples of interest, such as thin films, membranes, and microfluids, microresonators of various structures have been used. Such microresonators, including flat helical coils $[14,15]$ and stripline resonators $[16,17]$, as well as solenoids, have been summarized by Kentgens et al. [18]. Interestingly, the authors pointed out that the highest sensitivity per unit sample volume is attained not by the solenoid coil that is most widely used, but by a stripline resonator. In addition, they defined the limit of detection (LOD) as the number of spins that have to resonate in bandwidth of $1 \mathrm{~Hz}$ to give a signal-to-noise ratio of unity per scan. According to the their theoretical analysis, LOD for a stripline resonator, a flat helical coil, and a solenoid is 
$3 \times 10^{12}, 7 \times 10^{12}$, and $4 \times 10^{12}$, respectively, for proton resonance in a magnetic field of $14 \mathrm{~T}$ and a sample volume of $5 \mathrm{nl}$. These LOD values are so small (i.e., the expected sensitivity is so high) that use of microresonators for tiny systems that have been inaccessible should be feasible. Potential challenges may arise regarding sample handling, RF calibration, and parameter optimization.

\section{Realization of microcoil MAS}

\subsection{Piggyback microcoil MAS}

One approach for microcoil MAS takes a strategy of sticking a thin capillary sample tube on the rotor by fabricating a specially designed rotor cap [19]. Even though the microcoil is much smaller than the rotor, the thin sample tube sticking out of the rotor can be inserted into the microcoil, and the sample can be spun together with the rotor (Fig. 4a). This way of realizing microcoil MAS is known as the piggyback style [19]. It has been applied to ${ }^{1} \mathrm{H}-{ }^{13} \mathrm{C}$ CPMAS under very strong ${ }^{1} \mathrm{H}$ decoupling irradiation with intensities of up to $604 \mathrm{kHz}[19], 2 \mathrm{D}{ }^{13} \mathrm{C}$ correlation spectroscopy in a small sample [20], studies on MQ excitation and single-quantum (SQ) conversion efficiencies for ${ }^{23} \mathrm{Na}$ spins with very strong RF pulses of up to $940 \mathrm{kHz}$ [21], high-resolution ${ }^{1} \mathrm{H}$ NMR under homonuclear decoupling sequences and ${ }^{1} \mathrm{H}$-detected 2D heteronuclear correlation spectroscopy [22], determination of quadrupolar tensor values for half-integer spins in a rotating microcrystal [23], and ${ }^{13} \mathrm{C}-{ }^{13} \mathrm{C} 2 \mathrm{D}$ throughbond correlation experiments with a long decoherence time realized by strong ${ }^{1} \mathrm{H}$ decoupling and a spin-echo scheme [24].

The recent trend towards rotor downsizing to obtain faster spinning speeds 
has led to the use of small coils in commercial MAS probes. At the time of writing this review, the tiniest commercial MAS rotor has a diameter of 0.75 $\mathrm{mm}$, which realized a maximum attainable spinning speed of $110 \mathrm{kHz}$. Even though the precise size of the coil embedded in this $0.75-\mathrm{mm}$ spinning module is not clear, the coil volume would presumably be approximately $1 \mu \mathrm{l}$; the coil may be grouped into the microcoil, according to its original definition.

The spinning capability of the $0.75-\mathrm{mm}$ MAS probe drastically outperforms that of the home-built microcoil MAS probes reported so far. Nevertheless, the significance of the latter remains to be demonstrated for studies of different targets. Standard commercial MAS probes are designed for either fast spinning with small-volume rotors or slow spinning with large-volume rotors. However, for some biological applications, the spinning should be slow enough to avoid damage to the sample due to the centrifugal force, as pointed out by Wong et al. [25,26], who demonstrated slow spinning of microscopic biological samples with high sensitivity. It should also be mentioned that home-built microcoils can be an order of magnitude smaller than the smallest commercial microcoils.

\section{2 $M A C S$}

Sakellariou et al. [27] proposed another remarkable idea for realizing microcoil MAS, whereby a tiny resonant circuit composed of a microcoil and a chip capacitor is placed inside the rotor, and the microcoil is spun together with the rotor, as schematically described in Fig. 4b. RF signal transmission can be performed inductively and wirelessly, and its efficiency is as good as if the wire were physically tethered. In this strategy, termed magic-angle coil spinning (MACS), the microcoil can be wound on the capillary sample tube, 
so that the filling factor can be maximized.

The idea of wireless coupling of NMR or MRI coils is not of recent origin. As summarized by Hoult and Tomanek [28], this approach was already used a couple of decades ago for cases in which a physical connection between wires was impractical or the size of the sample of interest was too small compared to the measurable volume of the equipment available [29]. Such cases include in vivo spectroscopy, high-pressure NMR, MRI of small animals in a whole-body scanner [30], microfluidic systems [31], surgical implantation of a resonator inside the body of a living organism [32,33], stent antennas [34,35], simultaneous microimaging of multiple samples [36], and ${ }^{23} \mathrm{Na} \mathrm{NMR}$ and MRI [37]. In addition, a contactless mobile RF coil for switched angle spinning (SAS) has been reported [38-40]. In this approach, a doubly tuned saddle coil is capacitively coupled to the primary circuit of the probe. In contrast to previous SAS probes using sliding contacts or flexible leads, the wireless contact between the mobile and static parts of the circuit yields reliable RF transmission over a number of hops of the spinning axis, making long-term operation feasible.

Rotation of the resonator together with the sample is a revolutionary feature of MACS. Coil spinning at several $\mathrm{kHz}$ or more is not only impressive, but also beautifully solves the problem of inhomogeneous field distortion due to the bulk magnetic susceptibility of the coil wire [27,41], which is particularly serious for microcoil experiments in which the sample is spatially close to the wire. ${ }^{1} \mathrm{H}$ MAS, ${ }^{29} \mathrm{Si} \mathrm{MAS}$, and ${ }^{23} \mathrm{Na}$ MQMAS experiments in small-volume samples were demonstrated using the MACS scheme [27]. Wong et al. performed ${ }^{1} \mathrm{H}$ MACS in bovine tissue and human gastric mucosa [25,26].

Extension of MACS to ${ }^{1} \mathrm{H}_{-}{ }^{13} \mathrm{C}$ CP-MAS and ${ }^{13} \mathrm{C}-{ }^{13} \mathrm{C}$ two-dimensional corre- 
lation spectroscopy has recently been reported by Inukai and Takeda, who fabricated a MACS insert resonating at two separate frequencies [41]. Doubleresonance experiments were also demonstrated using a singly ${ }^{1} \mathrm{H}$-tuned MACS insert and a doubly tuned MAS probe. Aguiar et al. performed ${ }^{1} \mathrm{H}_{-}{ }^{13} \mathrm{C}$ cross polarization [42], and Wong et al. reported ${ }^{1} \mathrm{H}$-detected ${ }^{43} \mathrm{Ca}$ MACS experiments [43].

The most important practical aspect of the MACS scheme is that no hardware modification of the probe is required, so that a standard popular CPMAS probe can be used as is to perform microcoil MAS, unlike the other approach mentioned above. Thus, MACS could possibly be a versatile strategy for microcoil MAS when MACS resonators become readily available for various rotor sizes and resonance frequencies. At present, no commercial MACS insert is available. Jacquinot and Sakellariou summarized strategies for calculating and optimizing the electrical parameters, which is helpful for home-built equipment [44]. They estimated the LOD to be $3 \times 10^{12}$ spins for proton resonance at $500 \mathrm{MHz}$ using a MACS resonator of $0.1 \mathrm{~mm}$ in diameter and 0.2 $\mathrm{mm}$ in length. A possible problem in MACS of the effect due to the eddy current induced by spinning metal material in a strong static magnetic field was studied extensively by Aguiar et al., who also provide strategies for temperature stabilization [45]. Since the eddy current decreases with the amount of the metal spinning in the magnetic field, the heating effect decreases with coil miniaturization. It is worth mentioning that soldering is better avoided in fabrication of the MACS insert to avoid adding any extra metal. Instead, spot welding works well [41]. Wire bonding represents another solution [46]. 


\section{Disk MAS}

In both piggyback microcoil MAS and MACS, the sample container is a cylindrical capillary tube made of fused silica, quartz, or Vespel for target samples of powders, microcrystals, and biological tissues. Other small-volume samples of chemical interest include thin films, deposited layers, and membranes. Characterization of such "flat" samples is often important in material sciences and biochemistry. In many cases, non-destructive sample measurement is desirable so that the underlying chemical processes can be traced. With the aim of achieving non-destructive, high-resolution studies of flat samples, Inukai et al. investigated MAS of a disk-shaped, thin sample [47]. The scheme, referred to as disk MAS, is similar to piggyback microcoil MAS in that the sample is placed on top of a conventional rotor (Figs. 4c and 5a). Using a 4-mm spinning module, they examined the spinning speeds attainable, which were 4.2 and 7.0 $\mathrm{kHz}$ for disk diameters of 7 and $12 \mathrm{~mm}$, respectively (Fig. 5b). The 7-mm disk could spin at nearly the maximum spinning speed $(15 \mathrm{kHz})$ of the unmodified module. Roughly, the maximum spinning speed was limited by the velocity at the circumference of the spinning disk to slightly less than the speed of sound. Fig. $5 \mathrm{c}$ shows ${ }^{27} \mathrm{Al}$ disk MAS in which a $2-\mu \mathrm{m}$ layer of $\mathrm{Al}$ deposited on a 7 -mm-diameter substrate was spun at $7.5 \mathrm{kHz}$. Interestingly, the disk MAS spectrum shows a different shift from that obtained in a bulk $\mathrm{Al}$ sample measured using the conventional MAS scheme (Fig. 5d).

An important feature of disk MAS is that it opened the way to non-destructive, high-resolution characterization of thin samples. Inukai et al. demonstrated $e x$ situ ${ }^{7} \mathrm{Li}$ disk MAS analysis of a sputtered sample of $\mathrm{LiCoO}_{2}$, measuring ${ }^{7} \mathrm{Li}$ MAS spectra of an identical sample that was repeatedly annealed in a furnace 
[47]. They successfully observed a change in the ${ }^{7} \mathrm{Li}$ resonance line shape, ascribing it sample transition from an amorphous to a crystalline phase.

In disk MAS, a flat solenoid coil that just fits the spinning disk sample is used. An interesting point is the similarity of the coil-sample configuration to that of the case in Fig. 2, in which EMF was induced by a precessing nuclear magnetization distributed over a flat disk.

\section{Harnessing strong RF irradiation}

Strong RF irradiation using microcoils has been reported in several papers. Yamauchi et al. reported ${ }^{1} \mathrm{H}$ and ${ }^{27} \mathrm{Al}$ nutation frequencies of 4.7 and $3 \mathrm{MHz}$, respectively, demonstrating wide-line spectral excitation of ${ }^{27} \mathrm{Al}$ spins in singlecrystal and powder samples of sapphire [48]. More recently, Hagaman et al. reported generation of an $\mathrm{RF}$ field of $25 \mathrm{MHz}$ for ${ }^{19} \mathrm{~F}$ [49]. Such strong RF irradiation has allowed operations that could not be tested before. These include strong ${ }^{1} \mathrm{H}$ decoupling, MQ excitation and conversion of half-integer quadrupolar nuclei using intense pulses, and wide-line spectral excitation.

\subsection{Heteronuclear ${ }^{1} \mathrm{H}$ decoupling}

Janssen et al. observed ${ }^{13} \mathrm{C}$ CPMAS signals in uniformly ${ }^{13} \mathrm{C}$-labeled trialanine in $14.1 \mathrm{~T}$ under microcoil MAS at $10 \mathrm{kHz}$, and examined the height of the $\mathrm{C}_{\alpha}$ peak for various $\mathrm{CW}{ }^{1} \mathrm{H}$ decoupling intensities of up to $\nu_{1 \mathrm{H}}=604 \mathrm{kHz}$ [19]. Compared to the ${ }^{13} \mathrm{C}$ resonance line obtained at $\nu_{1 \mathrm{H}}=109 \mathrm{kHz}$, which is attainable using conventional CPMAS probes, further resonance line narrowing was found by increasing $\nu_{1 \mathrm{H}}$ to the regime only accessible using a microcoil. Inter- 
estingly, the peak intensity continued to increase until the decoupling strength reached ca. $500 \mathrm{kHz}$, at which a plateau was observed. This work demonstrated the importance of microcoil MAS in the study of spin decoupling dynamics.

To answer the question of whether this is the ultimate $\mathrm{CW}{ }^{1} \mathrm{H}$ decoupling performance that can be attained, it is necessary to consider line-broadening sources other than ${ }^{1} \mathrm{H}-{ }^{13} \mathrm{C}$ dipolar interactions. These include the bulk susceptibility effect and structural disorder. In addition, an effect that becomes significant with increasing decoupling field should be taken into account; nonresonant RF fields, such as the counter-rotating component of the oscillating field and decoupling RF irradiation, cause a shift of the peak position of the spins being observed. This shift, called the Bloch-Siegert effect [50-52], is proportional to the square of the irradiation intensity. If the RF amplitude is perfectly uniform over the sample volume, it merely causes an overall shift of the resonance line, from which the RF intensity can be accurately calibrated [53]. In the presence of RF inhomogeneity, however, the Bloch-Siegert shift causes broadening, as reported by McCoy and Mueller for frequency-selective homonuclear decoupling [54] and by Vierkötter for ${ }^{1} \mathrm{H}$ dipolar-decoupled ${ }^{19} \mathrm{~F}$ MAS NMR [55]. In conventional experiments with moderate RF intensities, the Bloch-Siegert effect is negligibly small, unless the irradiation frequency is relatively close to the observing frequency.

For very strong decoupling RF intensities, the Bloch-Siegert shift can be appreciable, even in ${ }^{1} \mathrm{H}$ decoupled ${ }^{13} \mathrm{C}$ NMR. Using irradiation and observation frequencies $\omega_{\text {irr }}$ and $\omega_{\text {obs }}$, the shift is represented as [55]

$$
\frac{\left(\gamma_{\mathrm{obs}} B_{1}\right)^{2}}{\omega_{\mathrm{irr}}^{2}-\omega_{\mathrm{obs}}^{2}}
$$

where $\gamma_{\text {obs }}$ is the gyromagnetic ratio of the observing spin, and $B_{1}$ is the in- 
tensity of the field applied at the decoupling spin. Janssen et al. performed ${ }^{1} \mathrm{H}-{ }^{13} \mathrm{C}$ double-resonance experiments at $14.1 \mathrm{~T}[19]$. For the strongest decoupling intensity they implemented $\left(\nu_{1 \mathrm{H}} \sim 0.6 \mathrm{MHz}\right)$, a shift of the ${ }^{13} \mathrm{C}$ resonance by ca. $10 \mathrm{~Hz}$ is expected. Note that the effect is proportional to the square of the RF amplitude. When the decoupling intensity is doubled to ca. 1.2 $\mathrm{MHz}$, which is still feasible with microcoil MAS, the shift would be four times greater.

The RF homogeneity can be improved by restricting the sample volume at the cost of sacrificing sensitivity. Assuming that a realistic compromise results in RF inhomogeneity of, say, approximately $10 \%$, broadening due to the BlochSiegert effect amounts to the order of hertz. Line broadening would become more prominent in lower static fields, as the shift is inversely proportional to the resonance frequency. Fig. 6 shows results for the dependence of the ${ }^{13} \mathrm{C}$ peak height of the methyl carbon in ${ }^{13} \mathrm{C}$-labeled L-alanine on the ${ }^{1} \mathrm{H}$ decoupling intensity for microcoil MAS experiments performed in a magnetic field of 7 $\mathrm{T}$ with a spinning speed of $7.5 \mathrm{kHz}$. The minimum line width was obtained for ${ }^{1} \mathrm{H}$ decoupling of ca. $600 \mathrm{kHz}$. The stronger irradiation caused the BlochSiegert broadening that is no longer negligible, leading to lower peak heights than expected.

Presumably, studies of spin decoupling dynamics by microcoil MAS may be of interest for RF intensities of up to several hundred kilohertz. Stronger decoupling than that would require techniques to compensate the Bloch-Siegert effect. When the isotropic shift is not of importance, the spin-echo scheme may be used [24]. Another way to suppress the Bloch-Siegert effect is to use an additional RF channel with a frequency corresponding to the mirror image of the irradiation frequency with respect to the Larmor frequency of the 
spins being observed [56-58]. It should also be mentioned that spin-decoupling dynamics under such strong irradiation becomes academic for heat-sensitive biological samples.

The ${ }^{1} \mathrm{H}$ decoupling sequences that are widely used nowadays, such as TPPM, SPINAL, and XiX, use phase modulation of the irradiating RF signal. In the context of microcoils, their performance for unconventionally large RF amplitudes was studied by Kentgens et al., who compared $\mathrm{CW}{ }^{1} \mathrm{H}$ decoupling with an RF amplitude of $350 \mathrm{kHz}$ and XiX decoupling with the same RF intensity [18]. Vasa et al. examined ${ }^{13} \mathrm{C}$ decoherence times using the spin-echo technique under TPPM and SPINAL decoupling sequences with a $400-\mathrm{kHz}$ irradiation amplitude [24]. As is the case for moderate decoupling intensities, these newer techniques worked better than traditional $\mathrm{CW}$ decoupling with the same RF power. The effect of Bloch-Siegert broadening in the presence of phase modulation is an open question. In addition, the larger the RF amplitude, the smaller is the pulse width. This leads to another technical challenge in the implementation of the sequence. We discuss this point in Section 7, as it is not restricted to decoupling but is relevant for strong RF pulses in general.

\section{2 $M Q$ excitation and $S Q$ conversion in $M Q M A S$}

In MQMAS experiments of half-integer quadrupolar nuclei, the sensitivity is affected by the initial population distribution over the zero-quantum eigenstates. In addition, important factors that determine the sensitivity are the efficiencies of creating MQ coherence and of converting the evolved MQ coherence into detectable SQ coherence. When the magnitude of the quadrupolar interaction $\nu_{\mathrm{Q}}$ is much greater than the RF intensity $\nu_{1}$, which is commonly 
the case for many current applications using conventional MAS probes, the strongest possible RF pulse is recommended to maximize the excitation and conversion efficiencies.

This is, however, not the case when $\nu_{1} \gg \nu_{\mathrm{Q}}$. For microcoil MAS, this condition arises for nuclei with relatively weak quadrupolar interactions. Since MQ excitation and SQ conversion processes are coherent evolutions of the density matrix, the optimal intensity and width of the pulse can be derived by calculation. Inukai and Takeda applied the strong RF approximation to the established formula for MQ excitation of a spin-3/2 nucleus, showing that the triple-quantum coherence is expected to show oscillation at a frequency that coincides with the nutation frequency $\nu_{1}$ [21]. In addition, the oscillation is amplitude-modulated, with a profile given by $\left(1-\cos \left(2 \pi \nu_{\mathrm{Q}} t\right)\right)$.

To verify this prediction, we performed microcoil MAS NMR of ${ }^{23} \mathrm{Na}$ for a polycrystalline sample of sodium sulfite. This revealed an unfavorable effect of RF inhomogeneity, which we estimated to be ca. $15 \%$ in this case. That is, the MQ excitation efficiency is proportional to $\sin \left(2 \pi \nu_{1} t\right)\left[1-\cos \left(2 \pi \nu_{\mathrm{Q}} t\right)\right]$. Since $\nu_{1} \gg \nu_{\mathrm{Q}}$, the former term needs to oscillate many times until the latter term reaches its maximum at $t=1 /\left(2 \nu_{\mathrm{Q}}\right)$. However, distribution of the $\nu_{1}$ value over the sample volume due to RF inhomogeneity causes destructive interference, leading to less efficient MQ excitation (Fig. 7a). It follows that in practice, much stronger RF intensity than the magnitude of the quadrupolar interaction would not be suitable for MQ excitation, unless the RF field is fairly homogeneous.

By contrast, the approximate formula for the SQ conversion efficiency valid for the case of $\nu_{1} \gg \nu_{\mathrm{Q}}$ is given by $\left[1-\cos \omega\left(2 \pi \nu_{\mathrm{Q}} t\right) \cos \left(4 \pi \nu_{1} t\right)\right]$, which is 
optimal at approximately $t=1 /\left(4 \nu_{1}\right)$. Since the first maximum occurs at only a quarter of the nutation period, the interference effect is small, even in the presence of considerable RF inhomogeneity (Fig. 7b).

\section{Coping with RF transients}

Application of very short pulses is limited by the hardware specification. In a study in which a $25-\mathrm{MHz}$ nutation rate was demonstrated, Hagaman et al. claimed that the $\pi / 2$ pulse width of $10 \mathrm{~ns}$ is "less than the minimum time interval of the spectrometer" [49]. However, even if the spectrometer (and the power amplifier) were capable of generating a 10-ns pulse, another obstacle would arise because of what is known as the pulse transient effect. In their work, the microcoil was impedance-matched at $376.5 \mathrm{MHz}$ with a $Q$ factor of 10.9. Then the time constant of the leading and trailing edges is expected to be $2 \times 10.9 /\left(2 \pi \times 376.5 \times 10^{6}\right)$, which is approximately $9 \mathrm{~ns}$. Since a full increase in the pulse amplitude takes approximately five times the time constant, mere transmission of the pulse signal to the tank circuit would not work; the pulse amplitude would begin to decrease before reaching the intended value.

To cope with this problem, the idea of generating a pre-emphasized RF profile, heuristically conceived by Hoult in 1970s and formulated very recently by our group, can be a solution. We provided a framework to calculate the shape of the pulse $a(t)$ back from the target pulse shape $b(t)$ [59], according to which

$$
a(t) \propto b(t)+\tau \frac{\mathrm{d}}{\mathrm{d} t} b(t)
$$

where $\tau=2 Q / \omega_{0}$ is the time constant of the tank circuit. The important implication of Eq. (15) is that the amplitude profile $a(t)$ of a pulse generated 
by a spectrometer should include, in addition to the target profile $b(t)$ itself, a correcting term given by its time derivative with a weighting factor determined by the time constant $\tau$. Note that the significance of the additional term increases with the rate of change of the pulse profile and with the time constant. Examples of active compensation of amplitude transients of RF pulses are shown in Fig. 8.

It should also be mentioned that the pulse amplitude has to be updated during a time interval that is much shorter than the time constant $\tau$ to gain control over the transient effect. In the aforementioned case of a nutation rate of $25 \mathrm{MHz}$, an amplitude-transient-compensated 10-ns pulse would require a spectrometer time resolution of $\sim 1 \mathrm{~ns}$. Considering that the minimum pulse width of the current state-of-the-art spectrometer is an order of magnitude greater than this, the specification appears to be formidable. Nevertheless, it should be feasible with current electronics technology. Presumably, such fine time resolution has been unnecessary so far, whereas the recent introduction of microcoils to realize very strong RF irradiation has brought new demands on NMR spectrometers.

Pulse design according to Eq. (15) works for amplitude transients, but not for phase transients, which can have a significant effect in multiple-pulse experiments using strong RF fields. The spin dynamics can differ from what is expected if it were not for phase transients. Separate studies on robustness against the transient effect will be necessary for individual sequences.

The idea of designing pre-emphasized shaped RF pulses was extended by Tabuchi et al. to actively compensate both amplitude and phase transients [60]. Since this approach realizes accurate RF pulse shapes without reducing 
the $Q$ factor of the tank circuit of the probe, it can be used not only to suppress the transient tail of the RF pulse, but also as a general concept for accurate RF pulsing, especially for multiple-pulse experiments. Another approach for suppressing the phase transient effect, as reported by Weber et al. [61], is to use multiple reflection of the RF pulse by intentionally inserting a point of impedance mismatch between the pulse source and the probe.

\section{Summary}

In the first part of this review, we analyzed the EMF induced across the terminals of a single-turn coil for a spatially distributed magnetization. It was shown that dilution of a small sample with magnetically inert matter increases the EMF. Dilution can thus be an option to increase the sensitivity if a small coil is not available. Next, we reviewed lithographic fabrication of microcoils and microresonators with various structures, focusing on the detection sensitivity. The highest sensitivity per unit sample volume is attained by a stripline resonator [18]. We described two strategies for realizing MAS of mass-limited samples, the piggyback and MACS schemes. In addition, we mentioned disk MAS, which allows MAS of small samples distributed over a thin layer. We then reviewed recent reports on microcoil solid-state NMR using strong RF irradiation that is only feasible with a microcoil. Wide-line spectral excitation, MQMAS, and spin decoupling are of particular interest.

Microcoil solid-state NMR has brought a number of interesting outcomes, as described above, which would make the field more exciting than ever. So far, however, microcoil experiments in solids have been reported by no more than several groups, whereas many groups have addressed microcoil NMR of fluids. 
One difficulty with solids is sample handling. For example, it is relatively difficult to pack powder samples into a thin capillary tube, whereas fluid samples can be easily transported. Indeed, samples can be automatically exchanged using a flow system. One noteworthy application of this is remotely detected NMR and MRI, as introduced by Moulé et al. [62], whereby spin polarization/encoding and detection are performed in physically different locations.

We also pointed out emerging problems regarding microcoils in solid-state NMR. These include line broadening due to the Bloch-Siegert shift, insufficient time resolution of current spectrometers, and pulse transients. These challenges should be viewed positively, as they imply that there is much room for further hardware and methodological progress, which should eventually extend the applicability of solid-state NMR to subjects beyond our present reach. Overall, microcoil solid-state NMR is still in the early stage of development and that is why it is currently an exciting field of research.

\section{Acknowledgment}

I thank Munehiro Inukai and Yasuto Noda for providing unpublished data from their experiments on microcoil MAS, MACS, and disk MAS experiments.

\section{References}

[1] T.L. Peck, R.L. Magin, P.C. Lauterbur, J. Magn. Reson. B108 (1995) 114-124.

[2] D.L. Olson, T.L. Peck, A.G. Webb, R.L. Magin, J.V. Sweedler, Science 270 (1995) 1967-1970. 
[3] D.I. Hoult, R.E. Richards, J. Magn. Reson. 24 (1976) 71-85.

[4] K.R. Minard, R.A. Wind, Concepts Magn. Reson. 13 (2001) 128-142.

[5] K.R. Minard, R.A. Wind, Concepts Magn. Reson. 13 (2001) 190-210.

[6] D.A. Seeber, J.H. Hoftiezer, W.B. Daniel, M.A. Rutgers, C.H. Pennington, Rev. Sci. Instrum. 71 (2000) 4263-4272.

[7] D.A. Seeber, R.L. Cooper, L. Ciobanu, C.H. Pennington, Rev. Sci. Instrum. 72 (2001) 2171.

[8] J.A. Rogers, R.J. Jackman, G.M. Whitesides, D.L. Olson, J.V. Sweedler, Appl. Phys. Lett. 70 (1997) 2464-2466.

[9] J. Dechow, A. Forchel, T. Lanz, A. Haase, Microelectron. Eng. 53 (2000) 517519.

[10] L.O. Sillerud, A.F. McDowell, N.L. Adolphi, R.E. Serda, D.P. Adams, M.J. Vasile, T.M. Alam, J. Magn. Reson. 181 (2006) 181-190.

[11] V. Demas, J.L. Herberg, V. Malba, A. Bernhardt, L. Evans, C. Harvey, S.C. Chinn, R.S. Maxwell, J. Reimer, J. Magn. Reson. 189 (2007) 121-129.

[12] V. Demas, A. Bernhardt, V. Malba, K. L. Adams, L. Evans, C. Harvey, R.S. Maxwell, J.L. Herberg, J. Magn. Reson. 200 (2009) 56-63. *Describes RF coils produced by laser lithography and compares their electrical properties with those of traditional wire-wound coils.

[13] H. Ryan, S.-H. Song, A. Zaß, J. Korvink, M. Utz, Anal. Chem. 84 (2012) 36963702.

[14] J. Dechow, T. Lanz, M. Stumber, A. Forchel, A. Haase, Rev. Sci. Instrum. 74 (2003) 4855. 
[15] C. Massin, F. Vincent, A. Homsy, K. Ehrmann, G. Boero, P.-A. Besse, A. Daridon, E. Verpoorte, N. de Rooij, R. Popovic, J. Magn. Reson. 164 (2003) $242-255$.

[16] P.J.M. van Bentum, J.W.G. Janssen, A.P.M. Kentgens, J. Bart, J.G.E. Gardeniers, J. Magn. Reson. 189 (2007) 104-113. *Describes a stripline probe with an in-depth analysis of the RF-field profile and the sensitivity.

[17] Y. Maguire, I. L. Chuang, S. Zhang, N. Gershenfeld, Proc. Natl. Acad. Sci. USA 104 (2007) 9198-9203. *Demonstrates one- and two-dimensional ${ }^{1} \mathrm{H}$ NMR in liquids using a planar microslot waveguide.

[18] A.P.M. Kentgens, J. Bart, P.J.M. van Bentum, A. Brinkmann, E.R.H. van Eck, J.G.E. Gardeniers, J.W.G. Janssen, P. Knijn, S. Vasa, M.H.W. Verkuijlen, J. Chem. Phys. 128 (2008) 052202. *Reviews microcoil detectors including helical, flat-helical, and stripline structures. The paper also summarizes their applications for ${ }^{75} \mathrm{As}$ wide-line excitation, ${ }^{13} \mathrm{C}-{ }^{13} \mathrm{C}$ correlation, ${ }^{1} \mathrm{H}$ heteronuclear decoupling, ${ }^{27} \mathrm{Al}$ MQMAS, thin-films, and microfluidics.

[19] H. Janssen, A. Brinkmann, E.R.H. van Eck, P.J.M. van Bentum, A.P.M. Kentgens, J. Am. Chem. Soc. 128 (2006) 8722-8723. *Demonstrates the piggyback strategy for microcoil MAS and studies ${ }^{1} \mathrm{H}-{ }^{13} \mathrm{C}$ heteronuclear decoupling dynamics using strong RF irradiation. ${ }^{1} \mathrm{H}$ CW decoupling efficiency is shown to increase with the ${ }^{1} \mathrm{H}$ nutation rate up to ca. $0.6 \mathrm{MHz}$.

[20] K. Yamauchi, T. Asakura, Chem. Lett. 35 (2006) 426-427. *Describes microcoil ${ }^{1} \mathrm{H}_{-}{ }^{13} \mathrm{C}$ CPMAS and two-dimensional ${ }^{13} \mathrm{C}$ RFDR experiments.

[21] M. Inukai, K. Takeda, Concepts Magn. Reson. B33 (2008) 115-123. *Studies on the efficiency of MQ excitation and SQ conversion in a ${ }^{23} \mathrm{Na}$ spin system under MAS for various RF intensities of up to ca. 0.94 MHz. Different RF intensity dependences of the MQ excitation and conversion efficiencies are discussed. 
[22] A. Brinkmann, S.K. Vasa, H. Janssen, A.P. Kentgens, Chem. Phys. Lett. 485 (2010) 275-280.

[23] S.K. Vasa, E.R.H. van Eck, J.W.G. Janssen, A.P.M. Kentgens, Phys. Chem. Chem. Phys. 12 (2010) 4813-4820. * Reports on ${ }^{1} \mathrm{H}$ homonuclear decoupling by FSLG and DUMBO and ${ }^{1} \mathrm{H}_{-}{ }^{13} \mathrm{C}$ HETCOR using microcoil MAS.

[24] S.K. Vasa, H. Janssen, E.R.H. van Eck, A.P.M. Kentgens, Phys. Chem. Chem. Phys. 13 (2011) 104-106. *Investigates the coherent lifetime of ${ }^{13} \mathrm{C}$ spins using the spin-echo technique under microcoil MAS and ${ }^{1} \mathrm{H}$ decoupling sequences of CW, TPPM, and SPINAL with strong irradiation. The authors show that the ${ }^{13} \mathrm{C}$ lifetime of the $\mathrm{CH}_{2}$ site in glycine exceeds $200 \mathrm{~ms}$ under SPINAL with a ${ }^{1} \mathrm{H}$ RF intensity of ca. $400 \mathrm{kHz}$, implying that the resonance line can be as narrow as ca. $5 \mathrm{~Hz}$ if other sources of broadening are eliminated.

[25] A. Wong, P.M. Aguiar, D. Sakellariou, Magn. Reson. Med. 63 (2010) 269-274.

[26] A. Wong, B. Jime, X. Li, E. Holmes, J.K. Nicholson, J.C. Lindon, D. Sakellariou, Anal. Chem. 84 (2012) 3843-3848. *Applies MACS for metabolic profiling of tissue samples.

[27] D. Sakellariou, G. Le Goff, J.-F. Jacquinot, Nature 447 (2007) 694-697. *Introduces MACS. This paper shows the feasibility of wireless signal transmission between a MAS probe and a spinning micorcoil at the magic angle, demonstrating ${ }^{1} \mathrm{H}$ and ${ }^{29} \mathrm{Si}$ MACS experiments.

[28] D. Hoult, B. Tomanek, Concepts Magn. Reson. 15 (2002) 262-285.

[29] P.L. Kuhns, M.J. Lizak, S.-H. Lee, M.S. Conradi, J. Magn. Reson. 78 (1988) $69-76$.

[30] H. Graf, P. Martirosian, F. Schick, M. Grieser, M.E. Bellemann, Med. Phys. 30 (2003) 1241-1245. 
[31] M. Utz, R. Monazami, J. Magn. Reson. 198 (2009) 132-136.

[32] M.D. Schnall, C. Barlow, V.H. Subramanian, J.S. Leigh Jr., J. Magn. Reson. 68 (1986) 161-167.

[33] N.A. Volland, T.H. Mareci, I. Constantinidis, N.E. Simpson, Magn. Reson. Med. 63 (2010) 998-1006.

[34] H.H. Quick, H. Kuehl, G. Kaiser, S. Bosk, J.F. Debatin, M.E. Ladd, Magn. Reson. Med. 48 (2002) 781-790.

[35] M. Busch, W. Vollmann, T. Bertsch, R. Wetzler, A. Bornstedt, B. Schnackenburg, J. Schnorr, D. Kivelitz, M. Taupitz, D. Grönemeyer, Magn. Reson. Med. 54 (2005) 775-782.

[36] T.A.O. Wang, L. Ciobanu, X. Zhang, A. Webb, Concepts Magn. Reson. B33 (2008) 236-243.

[37] J.A. Tang, A. Jerschow, Magn. Reson. Chem. 48 (2010) 763-770.

[38] C. Qian, A. Pines, R.W. Martin, J. Magn. Reson. 188 (2007) 183-189.

[39] I.M. Litvak, C.A. Espinosa, R.A. Shapiro, A.N. Oldham, V.V. Duong, R.W. Martin, J. Magn. Reson. 206 (2010) 183-189.

[40] C.A. Espinosa, P. Thureau, R.A. Shapiro, I.M. Litvak, R.W. Martin, Chem. Phys. Lett. 508 (2011) 314-319.

[41] M. Inukai, K. Takeda, J. Magn. Reson. 202 (2010) 274-278. *Extends MACS to double resonance, demonstrating ${ }^{1} \mathrm{H}^{13} \mathrm{C}$ double-resonance and $2 \mathrm{D}{ }^{13} \mathrm{C}-{ }^{13} \mathrm{C}$ correlation experiments using a doubly-tuned MACS insert.

[42] P.M. Aguiar, J.-F. Jacquinot, D. Sakellariou, Chem. Commun. 47 (2011) 21192121.

[43] A. Wong, P.M. Aguiar, T. Charpentier, D. Sakellariou, Chem. Sci. 2 (2011) 815. 
[44] J.-F. Jacquinot, D. Sakellariou, Concepts Magn. Reson. A38 (2011) 33-51. *Extensive study of coil sensitivity for various configurations of inductively coupled rotating microcoils.

[45] P.M. Aguiar, J.-F. Jacquinot, D. Sakellariou, J. Magn. Reson. 200 (2009) 6-14.

[46] V. Badilita, B. Fassbender, O. Gruschke, K. Kratt, R. Meier, D. Sakellariou, J. G. Korvink, U. Wallrabe, in: Proceedings of the 16th International Solid-State Sensors, Actuators and Microsystems Conference (TRANSDUCERS), 2011, pp. $2798-2800$.

[47] M. Inukai, Y. Noda, K. Takeda, J. Magn. Reson. 213 (2011) 192-195. *Introduces disk MAS, showing ${ }^{7} \mathrm{Li}$ NMR of a spinning thin layer of deposited battery material. The paper also demonstrates ex situ disk MAS measurements, tracing the heat-annealing process in the deposited layer.

[48] K. Yamauchi, J.W.G. Janssen, A.P.M. Kentgens, J. Magn. Reson. 167 (2004) 87-96. ${ }^{*}$ Implements wide-line excitation using a microcoil and applies it to ${ }^{27} \mathrm{Al}$ NMR in static single-crystal and powder samples.

[49] E.W. Hagaman, J. Jiao, T. Moore, J. Magn. Reson. 193 (2008) 150-152. *Presents ${ }^{19} \mathrm{~F}$ experiments using a microcoil and demonstrates a nutation rate of up to ca. $25 \mathrm{MHz}$.

[50] F. Bloch, A. Siegert, Phys. Rev. 57 (1940) 522-527.

[51] W.A. Anderson, Phys. Rev. 102 (1956) 151-167.

[52] W.A. Anderson, R. Freeman, J. Chem. Phys. 37 (1962) 85-103.

[53] R.F. Schulte, L. Sacolick, M.H. Deppe, M.A. Janich, M. Schwaiger, J.M. Wild, F. Wiesinger, NMR Biomed. 24 (2011) 1068-1072.

[54] M.A. McCoy, L. Mueller, J. Magn. Reson. 98 (1992) 674-679.

[55] S.A. Vierkötter, J. Magn. Reson. A118 (1996) 84-93. 
[56] S. Zhang, D.G. Gorenstein, J. Magn. Reson. 132 (1998) 81-87.

[57] S. Zhang, D.G. Gorenstein, J. Magn. Reson. 138 (1999) 281-287.

[58] H. Van Melckebeke, J.-P. Simorre, B. Brutscher, J. Magn. Reson. 170 (2004) $199-205$.

[59] K. Takeda, Y. Tabuchi, M. Negoro, M. Kitagawa, J. Magn. Reson. 197 (2009) 242-244. * Describes active compensation of the amplitude transient effect of RF pulses by designing a pre-emphasized shaped pulse that results in an intended pulse in the tuned coil.

[60] Y. Tabuchi, M. Negoro, K. Takeda, M. Kitagawa, J. Magn. Reson. 204 (2010) 327-332. *Extends active compensation of RF pulses to suppress phase transients as well as amplitude transients.

[61] J. Weber, M. Seemann, J. Schmedt Auf der Günne, Solid State Nucl. Magn. Reson. 43-44 (2012) 42-50.

[62] A.J. Moulé, M.M. Spence, S.-I. Han, J.A. Seeley, K.L. Pierce, S. Saxena, A. Pines, Proc. Natl. Acad. Sci. USA 100 (2003) 9122-9127. 


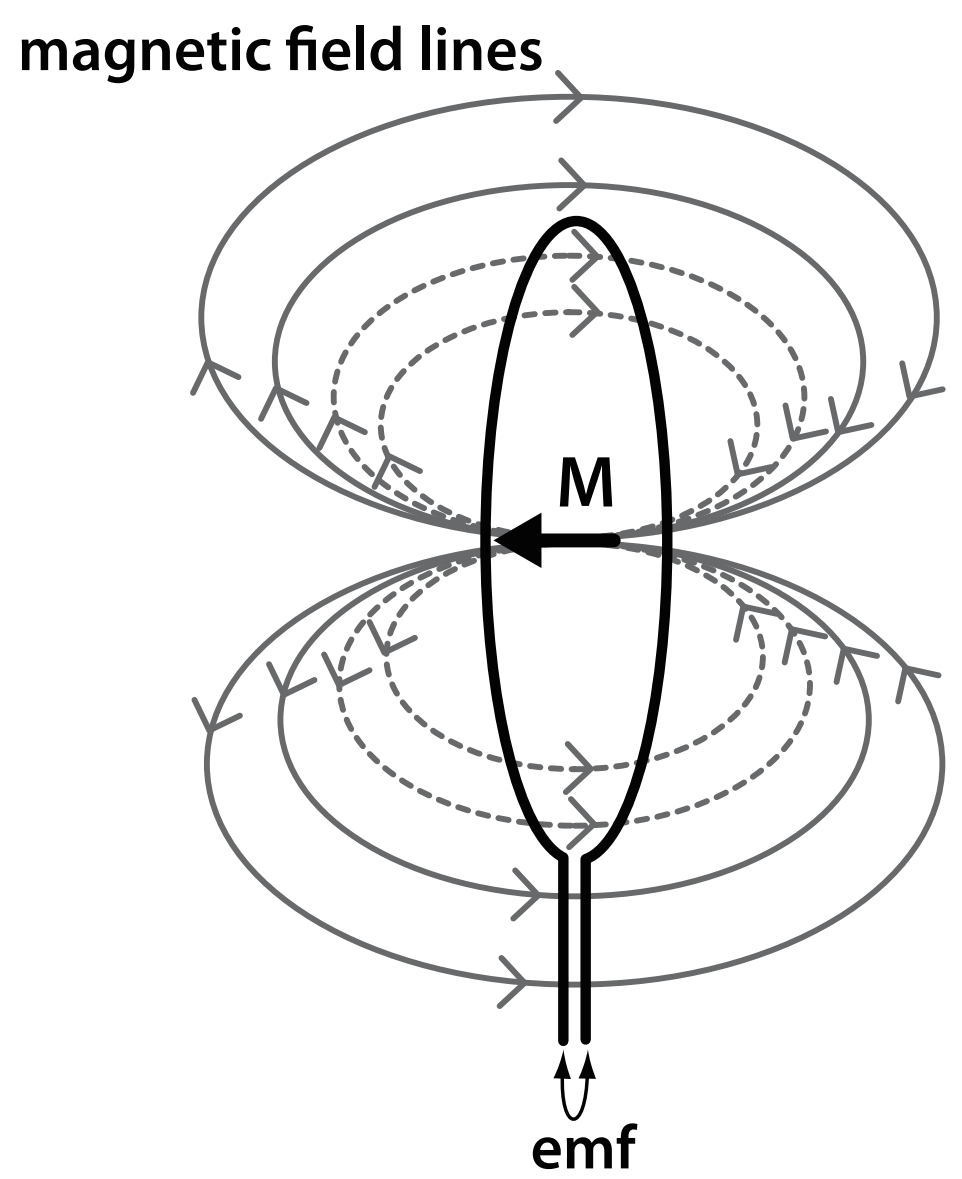

Fig. 1. A precessing magnetic moment $\mathbf{M}$ inside a loop of conducting wire induces electromotive force (EMF) across the wire terminals. The EMF is determined by a time derivative of the net magnetic flux penetrating through the loop. Magnetic field lines that are confined in the loop, as schematically depicted by the broken lines, make no contribution to the EMF. Only field lines that take a path that returns outside the loop, as described by the solid lines, can induce EMF. 
(a)

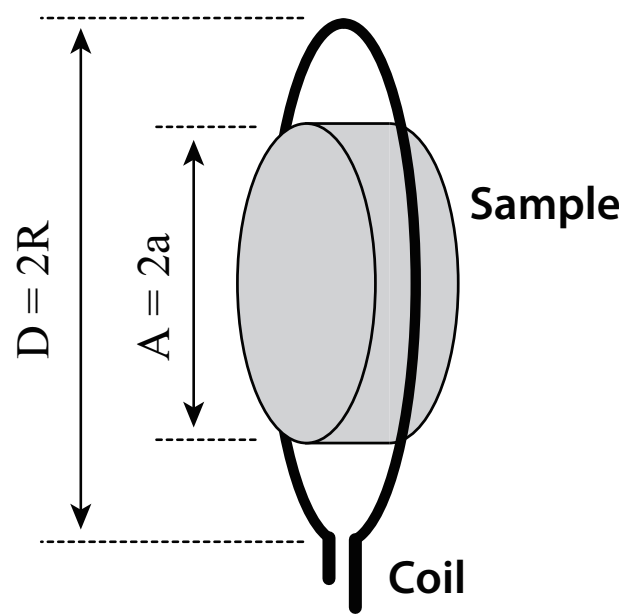

(b)

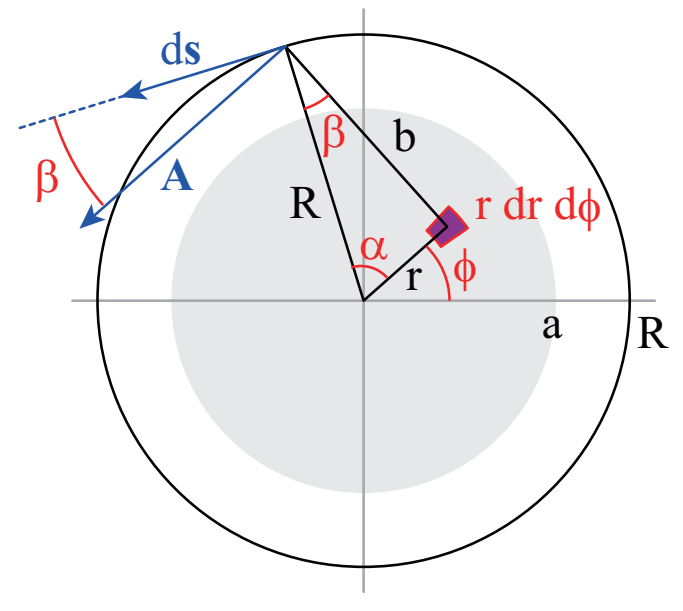

Fig. 2. (a) Schematic of a flat cylindrical sample inside a single-turn coil. (b) The vectors and angles used for calculation of the magnetic flux according to Eqs. (9)-(10).

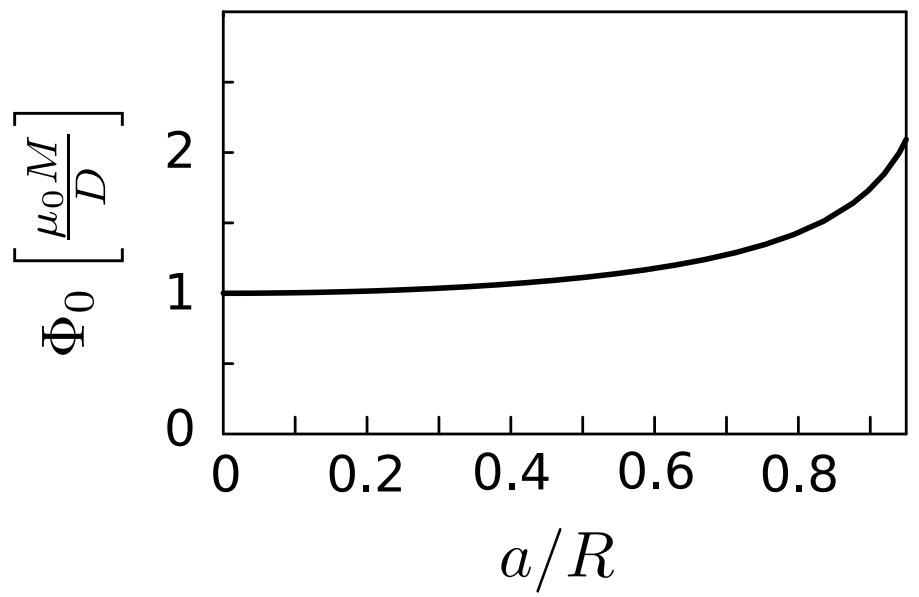

Fig. 3. Magnetic flux penetrating through a coil as a function of the ratio $a / R$ of the sample radius to the coil radius. The net magnetization is fixed. 
(a) Piggy-back

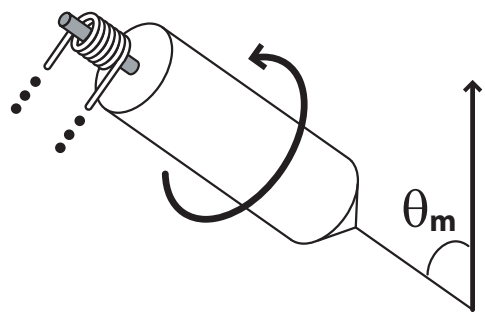

(b) MACS

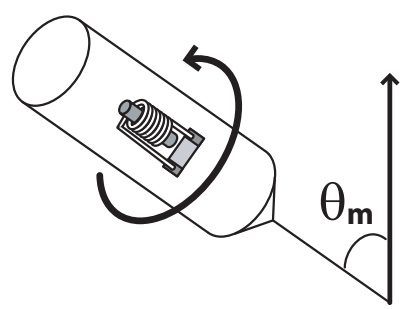

(c) disk MAS

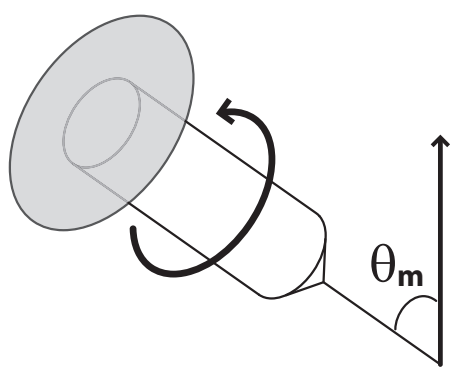

Fig. 4. Schematics of the strategies for MAS of small-volume samples. (a) Piggyback microcoil MAS, (b) magic-angle coil spinning (MACS), and (c) disk MAS.

(a)

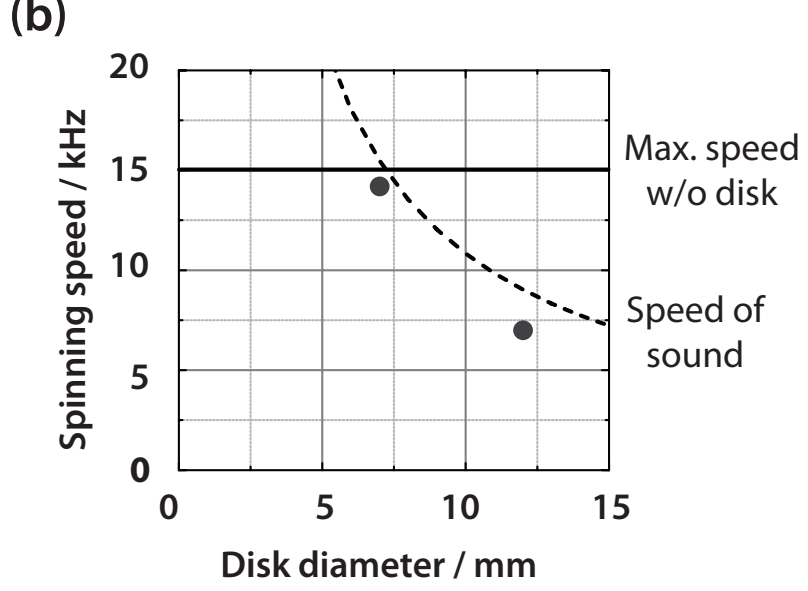

(c) vacuum-deposited Al

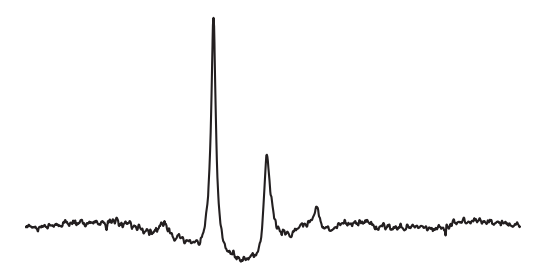

(d) bulk Al

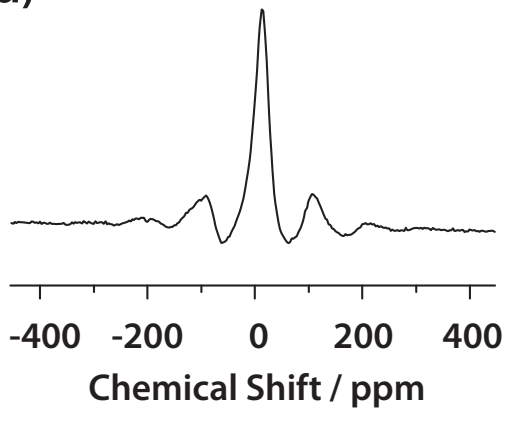

Fig. 5. (a) Sample attachment for a disk MAS experiment for a 4-mm rotor. (b) Spinning speeds attainable for the disk MAS apparatus reported in Ref. [47] (circles). The nominal maximum spinning speed of the 4-mm rotor is denoted by the solid line, and the spinning rate giving the speed of sound at the disk circumference by the broken line. (c) ${ }^{27} \mathrm{Al}$ disk MAS spectrum of a vacuum-deposited layer of $\mathrm{Al}$ obtained in a magnetic field of $7 \mathrm{~T}$. The thickness and diameter of the sample were 2 $\mu \mathrm{m}$ and $7 \mathrm{~mm}$, respectively. The spinning speed was $7 \mathrm{kHz}$. (d) ${ }^{27} \mathrm{Al}$ MAS spectrum of bulk Al powder. 


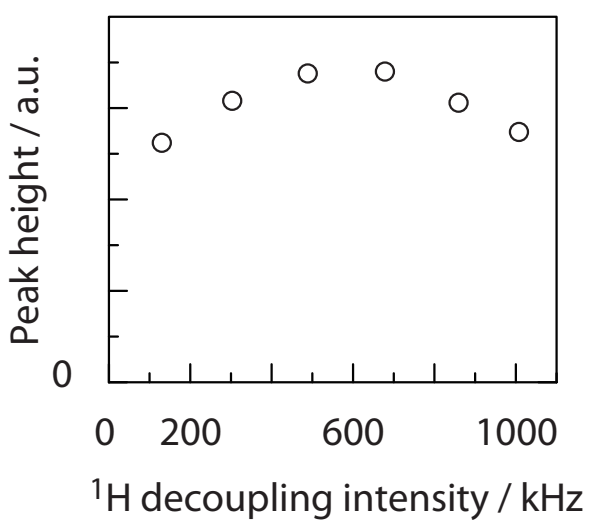

Fig. $6 .{ }^{13} \mathrm{C}$ peak heights of the methyl carbon in uniformly ${ }^{13} \mathrm{C}$-labeled L-alanine for various intensities of $\mathrm{CW}^{1} \mathrm{H}$ decoupling. Microcoil MAS experiments were performed in a magnetic field of $7 \mathrm{~T}$ with a spinning speed of $7.5 \mathrm{kHz}$. The inner diameter of the microcoil was $0.8 \mathrm{~mm}$. 
(a) MQ excitation
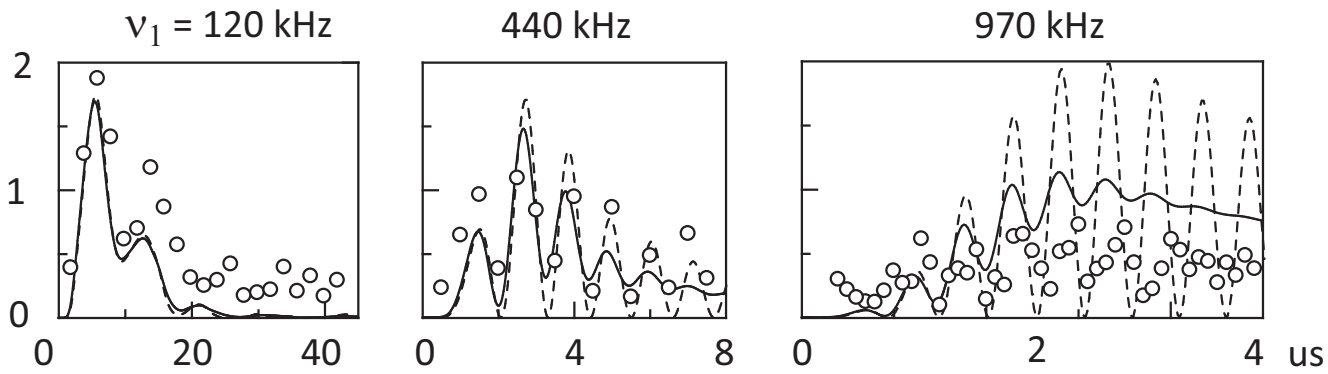

\section{(b) SQ conversion}
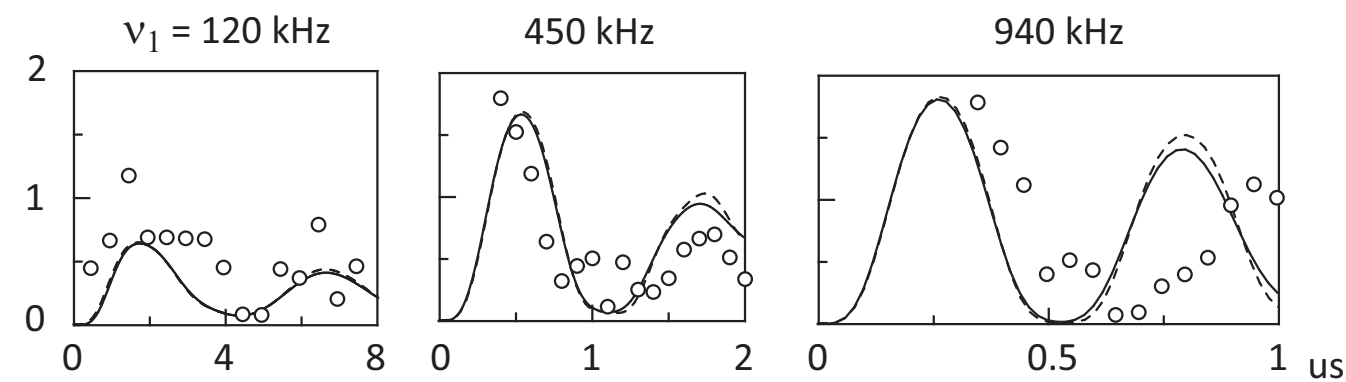

Fig. 7. (a) Triple-quantum excitation efficiencies of ${ }^{23} \mathrm{Na}$ spins in a polycrystalline sample of sodium sulfite as a function of the pulse width with RF intensities $\nu_{1}$ of (left) $120 \mathrm{kHz}$, (middle) $440 \mathrm{kHz}$, and (right) $970 \mathrm{kHz}$. (b) Pulse width dependence of single-quantum conversion efficiencies with $\nu_{1}$ of (left) $120 \mathrm{kHz}$, (middle) $450 \mathrm{kHz}$, and (right) $940 \mathrm{kHz}$. Circles represent measured values, while solid and broken lines denoted dependences calculated with and without taking 15\% RF inhomogeneity into account [21]. 


\section{Applied pulse Resultant pulse}

(a)

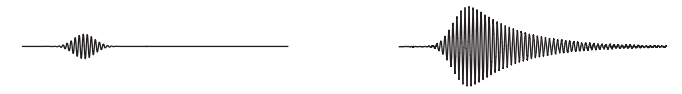

(b)
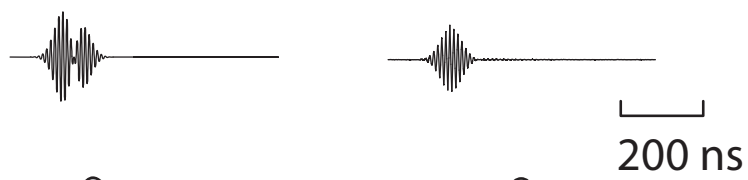

(c)
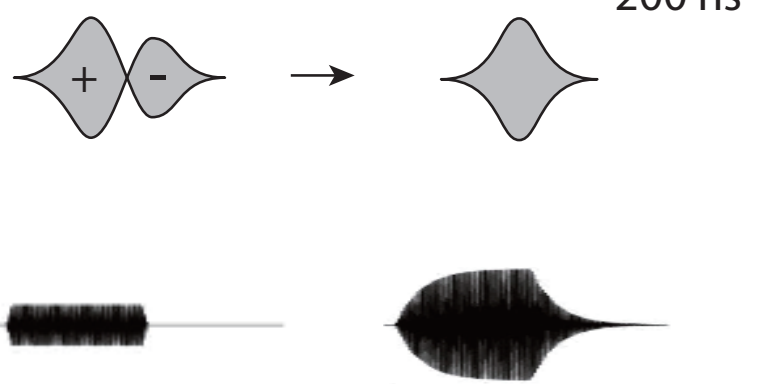

(d)
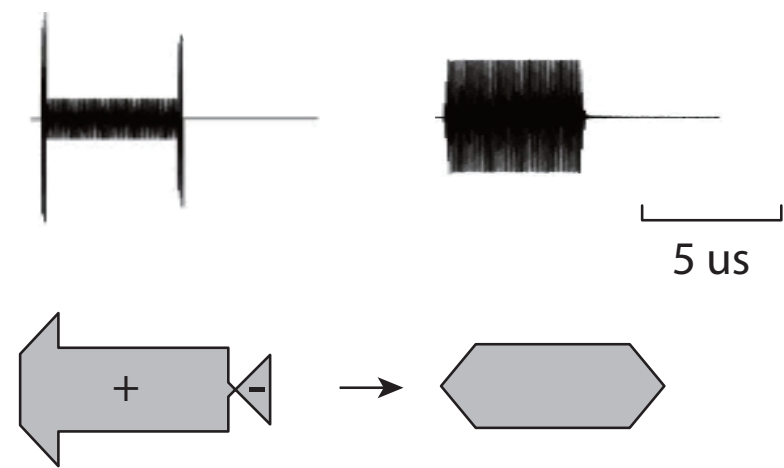

Fig. 8. Voltage profiles of RF excitation pulses generated by the transmitter of a spectrometer and the resulting RF fields monitored by a pickup coil placed inside the probes. $(\mathrm{a}, \mathrm{b})$ Waveforms of a Gaussian pulse and the pre-emphasized Gaussian pulse. The resonance frequency of the tank circuit was $102 \mathrm{MHz}$ and the $\mathrm{Q}$ factor was 40. (c,d) Waveforms of a trapezoidal pulse and actively compensated trapezoidal pulse. The resonance frequency and $Q$ factor were $12.7 \mathrm{MHz}$ and 35 , respectively. (a) and (b) are adapted from Ref. [59]. 